\title{
Molecular markers of anti-malarial drug resistance in Central, West and East African children with severe malaria
}

\author{
Christian N. Nguetse ${ }^{1}$, Ayola Akim Adegnika ${ }^{1,3}$, Tsiri Agbenyega ${ }^{4,5}$, Bernhards R. Ogutu 6 , Sanjeev Krishna ${ }^{1,7}$, \\ Peter G. Kremsner ${ }^{1,3}$ and Thirumalaisamy P. Velavan ${ }^{1,2,8,9^{*}}$
}

\begin{abstract}
Background: The Plasmodium falciparum multidrug resistance 1 (PfMDR1), P. falciparum Ca ${ }^{2+}$-ATPase (PfATP6) and Kelch-13 propeller domain (PfK13) loci are molecular markers of parasite susceptibility to anti-malarial drugs. Their frequency distributions were determined in the isolates collected from children with severe malaria originating from three African countries.

Methods: Samples from 287 children with severe malaria [(Gabon: $n=114) ;(G h a n a: n=89)$; (Kenya: $n=84)$ ] were genotyped for pfmdr1, pfatp6 and pfk 13 loci by DNA sequencing and assessing pfmdr 1 copy number variation (CNV) by real-time PCR.

Results: Pfmdr1-N86Y mutation was detected in 48, 10 and 10\% in Lambaréné, Kumasi and Kisumu, respectively. At codon 184, the prevalence of the mutation was 73\% in Lambaréné, 63\% in Kumasi and 49\% Kisumu. The S1034C and N1042D variants were absent at all three sites, while the frequency of the D1246Y mutation was 1, 3 and 13\% in Lambaréné, Kumasi and Kisumu, respectively. Isolates with two pfmdr 1 gene copy number predominantly harboured the N86Y wild-type allele and were mostly found in Kumasi $(10 \%)(P<0.0001)$. Among the main $p f m d r 1$ haplotypes (NFD, NYD and YFD), NYD was associated with highest parasitaemia $(P=0.04)$. At the pfatp6 locus, H243Y and A623E mutations were observed at very low frequency at all three sites. The prevalence of the pfatp6 E431K variant was 6, 18 and 17\% in Lambaréné, Kumasi and Kisumu, respectively. The L263E and S769N mutations were absent in all isolates. The pfk 13 variants associated with artemisinin resistance in Southeast Asia were not observed. Eleven novel substitutions in the pfk13 locus occurring at low frequency were observed.
\end{abstract}

Conclusions: Artemisinins are still highly efficacious in large malaria-endemic regions though declining efficacy has occurred in Southeast Asia. The return of chloroquine-sensitive strains following the removal of drug pressure is observed. However, selection of wild-type alleles in the multidrug-resistance gene and the increased gene copy number is associated with reduced lumefantrine sensitivity. This study indicates a need to constantly monitor drug resistance to artemisinin in field isolates from malaria-endemic countries.

Keywords: Malaria, P. falciparum, Pfmdr1, Pfatp6, Pfk13, Anti-malarial drugs, Resistance, Africa

\section{Background}

Malaria still claims hundreds of thousand deaths in subSaharan Africa, mostly children under the age of five and

\footnotetext{
*Correspondence: velavan@medizin.uni-tuebingen.de

1 Institute of Tropical Medicine, University Tübingen, Wilhelmstrasse 27,

72074 Tübingen, Germany

Full list of author information is available at the end of the article
}

pregnant women. The spread of artemisinin-resistant Plasmodium falciparum parasites from Southeast Asia to Africa is foreseen. Following the current World Health Organization (WHO) recommendation, artemisininbased combination therapy (ACT) is the first-line treatment of severe malaria [1]. Failure of ACT is well documented in Western Cambodia and the Thai-Myanmar border [2-4]. As a result, the WHO has developed 
strategies to contain the spread of resistant parasites to other countries [5].

It is clearly important to identify resistant phenotypes and constantly monitor for artemisinin resistance. Definitions of artemisinin resistance range from persistence of parasites on the third day after drug administration [6], an increased parasite clearance half-life [7, 8], a reduced parasite clearance rate $[3,9,10]$ and, finally, a treatment failure of ACT [11], although these are debated [11-14] and ACT failures also arise because of failure of partner drugs of artemisinins. The P. falciparum multidrug resistance 1 (PfMDR1) locus on chromosome 5 and the P. falciparum $\mathrm{Ca}^{2+}$-ATPase (PfATP6) locus on chromosome 1 genes may also modulate susceptibility of the parasites to artemisinins [15-18]. PfATP6, a candidate marker for artemisinin susceptibility $[16,19]$, is a sarcoendoplasmic reticulum calcium-ATPase (serca)-type calcium pump of the parasite suggested to be the target for artemisinins [15]. Increase in the gene copy number (copy number variation, CNV) of $p f m d r 1$ [20-22], which encodes an ATP-binding cassette transporter homologue of the P-glycoprotein 1 [23] is also involved in multidrug resistance, including to artemisinins [24]. Recently, a new locus was identified, the kelch protein located on chromosome 13 (PfK13 propeller); pfk13 mutations associated with clinical artemisinin resistance in Cambodia [25], and in vitro ring-stage survival rates [26], have now spread across Southeast Asia and South China [27, 28 ] and have shown to also arise independently [29]. For these reasons, mutations in the pfatp6, pfmdr1 and pfk 13 genes may be useful molecular markers of artemisinin resistance [25, 30-32].

This is a sub-study conducted by the "Severe Malaria in African Children" (SMAC) consortium [33]. The goal of this study was to describe parasite polymorphisms at the pfmdr1, pfatp 6 and pfk13 loci, including the pfmdr1 copy number in West, Central and East African children with severe malaria.

\section{Methods}

\section{Study design and sample collection}

Two hundred and ninety-six children aged $0.5-10$ years (120 in Lambaréné, Gabon, 90 in Kumasi, Ghana, 86 in Kisumu, Kenya) were randomly selected from the SMAC study [33]. Blood samples from all patients were collected in heparinized tubes. Storage and transport of specimens were done using a cold chain transport tool for subsequent molecular analyses.

\section{PfMDR1, PfATP6 and PfK13 genotyping}

Genomic DNA was isolated using the QIAamp DNA mini blood kit (Qiagen, Hilden, Germany). The pfmdr1 mutations N86Y, Y184F, S1034C, N1042D and D1246Y were screened by nested PCR using primer pairs described elsewhere [34]. The pfatp6 mutations H243Y, L263E, E431K, A623E and S769N were screened by PCR using the primer pairs designed by Zakeri et al. [35] Mutations M476I, Y493H, R539T, I543T and C580Y in the $p f k 13$ gene were screened using the primer pairs indicated by Ariey et al. [25]. In brief, $10 \mathrm{ng}$ of parasite genomic DNA were added to a $20 \mu \mathrm{L}$ reaction mixture

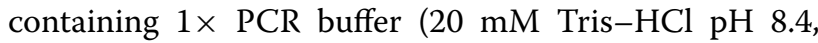
$50 \mathrm{mM} \mathrm{KCl}, 2.5 \mathrm{mM}$ of $\mathrm{MgCl}_{2}$ ), $0.125 \mathrm{mM}$ of dNTPs, $0.25 \mathrm{mM}$ of each primer and $1 \mathrm{U}$ Taq DNA polymerase (Qiagen, Hilden, Germany). The PCR reaction was run on a PTC-200 Thermal cycler (MJ Research, Waltham, USA). PCR products were visualized through electrophoresis on a $1.2 \%$ agarose gel stained with SYBR green I in $1 \times$ Tris-electrophoresis buffer $(90 \mathrm{mM}$ Tris-acetate, pH 8.0, 90 mM boric acid, $2.5 \mathrm{mM}$ EDTA). Subsequently, PCR products were purified (Exo-SAP-IT, USB, Affymetrix, Santa Clara, CA, USA) and directly used as templates for DNA sequencing using the BigDye terminator v. 1.1 cycle sequencing kit (Applied Biosystems, Foster City, USA) on an ABI 3130XL DNA sequencer. Polymorphisms were identified by assembling the sequences with the reference sequence of the pfmdr1 (NC_004326.1), pfatp6 (NC_004325.1) and pfk13 (NC_004331.2) genes using the Codoncode Aligner 4.0 software and visually reconfirmed from their electropherograms.

\section{PfMDR1 copy number}

The $p f m d r 1$ gene copy number was estimated by TaqMan real-time PCR using the hydrolysis probes as previously described [36]. In brief, $10 \mathrm{ng}$ of genomic DNA were added to a $25 \mu \mathrm{L}$ reaction mixture containing $1 \times$ TaqMan buffer [ $8 \%$ glycerol, $0.625 \mathrm{U}$ DNA polymerase, $5.5 \mathrm{mmol} / \mathrm{L} \mathrm{MgCl}_{2}, 300 \mu \mathrm{mol} / \mathrm{L}$ dNTPs, $600 \mathrm{nmol} / \mathrm{L}$ passive reference dye ROX (5-carboxy-X-rhodamine), $\mathrm{pH}$ 8.3], $300 \mathrm{nmol} / \mathrm{L}$ of each primer, $100 \mathrm{nmol} / \mathrm{L}$ of each probe, and $5 \mu \mathrm{L}$ of the template DNA. The real-time PCR reaction was run on a Corbett device (Research RG-3000, Qiagen, Hilden, Germany). The thermal conditions after a pre-incubation step $\left(95^{\circ} \mathrm{C}, 5 \mathrm{~min}\right)$ were 50 cycles of $95^{\circ} \mathrm{C}$ for $15 \mathrm{~s}$ and $58{ }^{\circ} \mathrm{C}$ for $1 \mathrm{~min}$. Genomic DNA from P. falciparum reference strain 3D7 was used as calibrator and $P$. falciparum $ß$-tubulin, a house-keeping gene, was used as reference gene. For multiple $p f m d r 1$ gene copy numbers, DNA from the Dd2 clone was used as control. The $2^{-\Delta \Delta \mathrm{Ct}}$ method of relative quantification was used to estimate the gene copy number [37]. Each sample was run in triplicate along with the reference DNA samples from clones 3D7 and Dd2, which are known to have a pfmdr1 gene copy number of 1 and 2-4, respectively. The mean and standard deviation of the three threshold cycle $(\mathrm{Ct})$ values were calculated for each sample. The 
experiment was repeated if one of the following results was obtained: $\Delta \Delta \mathrm{Ct}$ spread $>1.5$; $\mathrm{Ct}$ values $>35$; or copy number value $=1.3-1.6[36]$. Copy number estimates were rounded to the nearest integer and parasites with more than 1.5 copies were considered multiple copies [36]. A sample was considered to carry one copy of the gene if the $\mathrm{N}$-fold copy number was between 0.5 and 1.5 $(0.5<\mathrm{N}$-fold $<1.5)$.

\section{Statistical analysis}

Data were analysed using GraphPad Prism (GraphPad software Inc, La Jolla, CA). Mann-Whitney U and Kruskal-Wallis H One-way ANOVA tests were executed to determine possible associations of parasitaemia with the increased gene copy number and with the haplotypes of the genes investigated. Dunn's multiple comparison test was used to test for differences among study sites. The level of significance was set to a $P$ value of $<0.05$.

\section{Results}

In total, 296 children with severe malaria were enrolled in this study. Available for genetic analyses were 287 patients.

\section{PfMDR1 polymorphisms}

All 287 samples were successfully genotyped for $p f m d r 1$ mutations. The frequency distribution of genetic variants identified is given in Table 1. The S1034C and N1042D mutations were absent at all three sites, while the frequency of the D1246Y mutation was 1, 3 and 13\% in Lambaréné, Kumasi and Kisumu, respectively.

In Lambaréné, the prevalence of the wild-type N86Y allele was $40 \%$. The frequency of mixed genotypes was $12 \%$. At codon 184, the frequency of the wild-type and mutant Y184F alleles was 25 and $73 \%$, respectively, whereas the prevalence of mixed genotypes was $2 \%$. The double mutations N86Y/Y184F, and N86Y/D1246Y were observed in 51 (45\%) and 1 (1\%) samples, respectively.

In Kumasi, the majority of samples (73\%) carried the wild-type N86Y allele. The prevalence of mixed genotypes was $17 \%$. The prevalence of the wild-type Y184F allele was 30, and 7\% for the mixed genotypes. The prevalence of double mutations N86Y/Y184F, and N86Y/ D1246Y was 9 and $1 \%$, respectively. There was only one sample with the triple mutant N86Y-Y184F-D1246Y allele.

In Kisumu, $82 \%$ of samples carried the wild-type allele N86Y, and 8\% had mixed genotypes. The frequency of samples carrying the wild-type Y184F allele $44 \%$. The samples with mixed genotypes were $7 \%$. Two samples (2\%) had the double mutant N86Y/Y184F, and 4 (5\%) had the double mutant N86Y/D1246Y. One sample carried the triple mutant N86Y-Y184F-D1246Y allele.

\section{PfMDR1 copy number}

The copy number of the $p f m d r 1$ was successfully determined and results were available for 285 isolates. Two isolates, one each in Lambaréné and Kisumu, were removed from the analysis because of inconsistent results. The overall $p f m d r 1$ copy number mean was 0.89 (range 0.5-1.9), 1.2 (range 0.5-1.9) and 0.95 (range 0.51.7) in Lambaréné, Kumasi and Kisumu, respectively. With the control DNA, the results were reproducible with a mean copy number of 1.1 and a standard deviation of 0.18 for the 3D7 strain. The Dd2 control gave a mean copy number of 3.98 with a standard deviation of 0.38 . When rounded to an integer, three, nine and one isolates with two gene copy numbers were observed in Lambaréné, Kumasi and Kisumu, respectively. The values of the pfmdrl copy number from the three study sites are depicted in Fig. 1. When rounded to the nearest integer, 1 gene copy number was found in 97, 90 and 99\% isolates from Lambaréné, Kumasi and Kisumu, respectively. There was a significant difference of the $p f m d r 1$ copy number among the parasites from the three study sites $(P<0.0001)$. Dunn's multiple comparison showed that parasites retrieved in samples from Kumasi had more copy numbers than identified in those from Lambaréné and Kisumu. In this study, the $p f m d r 1$ copy number was not related to the baseline parasitaemia [Mean parasitaemia, Kisumu $=183,233$ (range 7452-1,677,780), Kumasi $=171,835$ (range 6240-1,209,000) and

Table 1 Frequency distribution of mutant pfmdr1 and pfatp6 alleles in isolates from Lambaréné, Kumasi and Kisumu

\begin{tabular}{|c|c|c|c|c|c|c|}
\hline \multirow[t]{3}{*}{ Site (N) } & \multicolumn{6}{|c|}{ Number (\%) of samples with allelic change at these codons } \\
\hline & \multicolumn{3}{|l|}{ Pfmdr1 } & \multicolumn{3}{|c|}{ Pfatp6 } \\
\hline & $86 \mathrm{Y}$ & $184 \mathrm{~F}$ & $1246 Y$ & $243 Y$ & $431 \mathrm{~K}$ & 623E \\
\hline Lambaréné (114) & $55(48)$ & $83(73)$ & $1(1)$ & $1(1)$ & $6(6)$ & $1(1)$ \\
\hline Kumasi (89) & $9(10)$ & $56(63)$ & $3(3)$ & $1(1)$ & $13(18)$ & $1(1)$ \\
\hline Kisumu (84) & $8(10)$ & $41(49)$ & $11(13)$ & $2(3)$ & $13(17)$ & $1(1)$ \\
\hline
\end{tabular}

There were no mutant alleles at the following codons: pfmdr1 S1034C, N1042D and pfatp6 L263E, S769N 


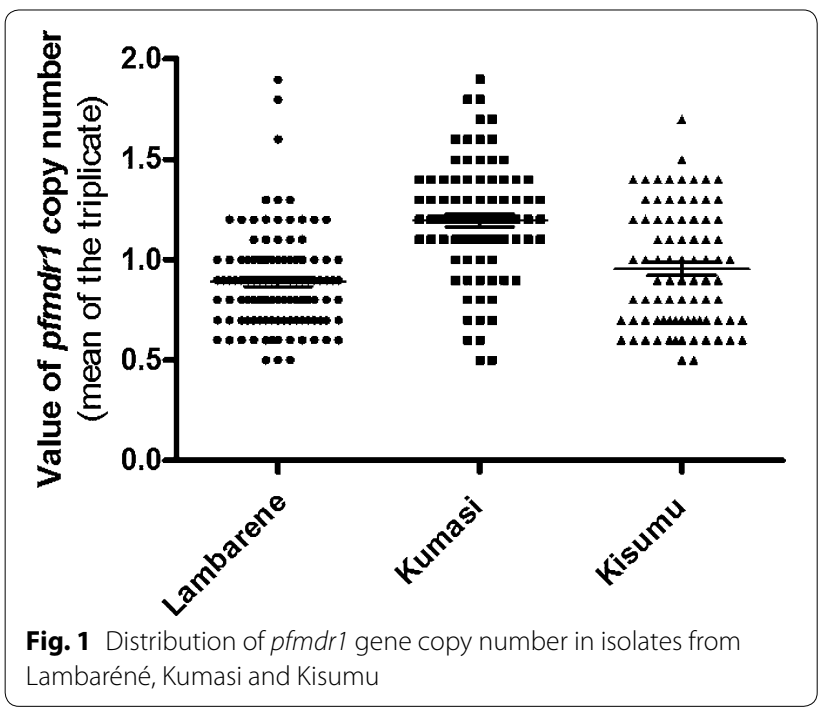

Lambaréné $=80,986$ (range 5376-745,100)]. The isolates with an increased copy number were found to harbour predominantly the N86Y wild-type allele alone (9/13) or combined with the mutant allele $(11 / 13)$ compared to the mutant allele N86Y alone (2/13).

\section{PfMDR1 haplotypes}

The $p f m d r 1$ haplotypes were reconstructed including the mutations N86Y, Y184F and D1246Y. The isolates with a mixture of two haplotypes were each counted as carrying both. In total, eight different haplotypes were observed. In Lambaréné, the haplotype YFD was the most prevalent one, occurring at a frequency of $57 \%$, followed by NFD (30\%), NYD (24\%), YYD (5\%) and YYY (1\%). In Kumasi, the most prevalent haplotype was NFD (60\%) followed by NYD (34\%), YFD (23\%), YYY (3\%) and NYY (2\%). The other haplotypes NFY, YYD and YFY all occurred at a frequency of $1 \%$. In Kisumu, the haplotype NFD was the most prevalent, occurring at a frequency of $51 \%$, followed by NYD (37\%), NYY and YFD both occurring at a frequency of $7 \%$, YYD and YYY each at 6\%, NFY (2\%) and YFY (1\%). The most prevalent haplotypes associated with increased pfmdr1 copy number were NFD, NYD and YFD occurring six, five and four times, respectively. For these main haplotypes (NFD, NYD and YFD), the mean parasitaemia was significantly different $(P=0.04)$ and this difference was observed between the haplotypes NYD and YFD $(P=0.012)$.

\section{PfATP6 polymorphisms}

Of the 287 collected samples, only 250 samples (99/114, 74/89 and 77/84 in Lambaréné, Kumasi and Kisumu, respectively) were successfully genotyped for pfatp6 mutations (Table 1). In the samples collected in
Lambaréné, the prevalence of the pfatp6 H243Y, E431K and A623E mutant alleles was 1, 6 and 1\%, respectively. The frequency of the mixed genotypes at codon 243 was $1 \%$. In Kumasi, two isolates harboured the mixed genotypes at codons 243 and 623 at a frequency of 1\% each, respectively. Thirteen (18\%) samples carried the mutant allele E431K while two samples (1\%), one each carried the mutant $\mathrm{H} 243 \mathrm{Y}$ and $\mathrm{A} 623 \mathrm{E}$ alleles, respectively. In Kisumu, the frequency of the mutant alleles E431K, $\mathrm{H} 243 \mathrm{Y}$ and A623E was 17, 3 and 1\%, respectively. The prevalence of the mixed genotypes only found at codon A623E was 3\%.

\section{PfATP6 haplotypes}

The pfatp6 haplotypes were reconstructed using the mutations H243Y, E431K and A623E. Isolates with a mixture of two haplotypes were counted as carrying both. Five haplotypes were investigated. The HEA haplotype was the most prevalent one, occurring at a frequency of 92,80 , and $81 \%$ in Lambaréné, Kumasi and Kisumu, respectively. In Lambaréné, the other haplotypes were HKA, HEE and YEA occurring at frequencies of 6,1 and $2 \%$, respectively. In Kumasi, the haplotypes HKA, HEE, YEA and YKA were found at frequencies of 18, 3, 1 and $1 \%$, respectively. In Kisumu, the haplotypes HKA, HEE and YEA were found at frequencies of 17, 4 and 3\%, respectively. There was no association of the mean parasitaemia with any of the haplotypes.

\section{PfK13 polymorphisms}

The nonsynonymous mutations M476I, Y493H, R539T, I543T and C580Y, which were previously shown to be associated with an increased parasite clearance half-life time in Cambodia were absent in these samples. However, several yet unrecognized variants were identified individually in eleven parasites (three, two and six in Lambaréné, Kumasi and Kisumu, respectively), albeit at very low frequencies (Table 2).

\section{Discussion}

In the absence of an effective malaria vaccine, treatment failures associated with ACT (TFACT) will hamper global efforts in reducing malaria mortality and morbidity. Besides studies on Africans, one of the most affected populations, highlighted the significant contribution of host genetics towards the stabilisation of these two indicators of health status [38-41]. A previous study has shown for the first time that the pfmdr1 N86Y polymorphism is associated with delayed parasite clearance when artesunate was used in monotherapy [33]. This finding may be of importance, as it demonstrates in vivo modulation of the efficacy of artesunate, the drug of choice against severe malaria. However, there is an important 
Table 2 Mutations in the Kelch-13 propeller domain (pfk13) of isolates from Lambaréné, Kumasi and Kisumu

\begin{tabular}{|c|c|c|c|c|c|}
\hline Codon position & Amino acid reference & Nucleotide reference & Amino acid mutation & Nucleotide mutation & Site \\
\hline 549 & S & tct & S & $\mathrm{tcC}$ & Kumasi \\
\hline 553 & $P$ & $c c \mathbf{g}$ & $P$ & $\mathrm{CCA}$ & Kisumu \\
\hline 578 & A & $\mathbf{g c t}$ & $S$ & $\mathbf{T} \subset t$ & Kisumu \\
\hline 589 & V & $\mathbf{g t c}$ & 1 & Atc & Lambaréné \\
\hline 589 & V & gtc & V & gtT & Lambaréné \\
\hline 639 & G & ggt & G & $g g C$ & Kumasi \\
\hline 645 & N & aac & N & aa $\mathbf{T}$ & Lambaréné \\
\hline 666 & V & gta & V & $\mathrm{gt} \mathbf{G}$ & Kisumu \\
\hline
\end{tabular}

need to clearly define artemisinin resistance owing to the large number of definitions found in the literature. Besides inclusive debates are rather welcome if the goal is to tackle the problem of multidrug-resistant parasites [12]. In the meantime, continuous monitoring of the molecular markers of anti-malarial drug resistance will help to prevent the spread of resistant strains and serve as basis for future update of the treatment policy.

Concerning the frequency distribution of the $p f m d r 1$ mutations, when comparing with previous studies [42], an increased prevalence of the wild-type allele N86Y was observed in Gabon. This allele is reported to be associated with exposure to the anti-malarial drug combination artemether-lumefantrine (AL) [43, 44]. The mutation N86Y has previously been associated with a lower 50\% inhibitory concentration (IC50) for artemisinin and dihydroartemisinin, as compared with the wild-type allele [18]. However, a recent study showed that the pfmdr1 N86Y variant was associated with prolonged parasite clearance in vivo [33]. Its high frequency in Lambaréné might be alarming but parasites in that region are still sensitive to artemisinins. Further studies assessing the sensitivity of these parasites over time are needed. In Kumasi, the findings reported here were consistent with the study published by KwansaBentum et al. [45]. In Kisumu, the prevalence of the pfmdr1 wild-type allele N86Y was higher compared to a previous study [46]. This allele has been associated in vitro with a three to fourfold increase of the lumefantrine IC50 values, as compared to the mutant N86Y allele [47], and suggested together with other pfmdr1 polymorphisms to be important determinants of parasite sensitivity to anti-malarials [48]. In addition, the $p f m d r 1$ wild-type allele N86Y is reported to be predominant in recurrent infections [49] and to increase in a fivefold degree the risk of recrudescence in individuals treated with AL [50]. These findings support the need of a constant monitoring of the parasite susceptibility to AL in Kumasi and Kisumu.
The mutations S1034C and N1042C, which have been reported to reduce parasite resistance to mefloquine [51] were not observed at all in this study. Regarding the variant D1246Y, a threefold increased prevalence from $4 \%$ [46] to $13 \%$ in Kisumu, compared to the lower prevalence of $\mathrm{D} 1246 \mathrm{Y}$ of $9 \%$ [42] to $1 \%$ in Lambaréné, and of $14 \%$ [52] to $3 \%$ in Kumasi was noted. Sidhu et al. [53] have reported that the combination of the mutant alleles S1034C-N1042D-D1246Y, which occurs frequently in South America, was associated with increased parasite susceptibility to artemisinin. However, in this study, S1034C and N1042C were absent, except D1246Y. This finding suggests that there is some selective pressure exerted by the parasite on the drug transporter $p f m d r 1$ gene at codons 1034 and 1042. The selection of the wildtype alleles following ACT use is worrying as a higher virulence of these wild-type genotypes has been linked to febrile illness [54].

PfMDR1 gene amplification has been reported to be selected by the use of mefloquine, artesunate, lumefantrine and quinine [36, 52, 55-57]. Uhlemann et al. [56] found $5 \%$ of $P$. falciparum isolates in Lambaréné carrying two copies of the $p f m d r 1$ gene, while 7 years later the same authors did not identify any isolates with multicopies. In the present study, $3 \%$ of the isolates were found carrying two copies. This indicates a possible circulation of parasites carrying several copies of the pfmdrl gene in Lambaréné at a low prevalence. The low frequency of isolates with pfmdr1 multicopies observed in Kisumu is consistent with previous studies [58,59]; and in Ghana, these findings are in agreement with previous work [52, 60]. The $p f m d r 1$ gene amplification is one of the best indicators for monitoring parasite resistance to treatment with artesunate mefloquine combination, and some but not all parasites with increased $p f m d r 1$ gene copies had a reduced sensitivity to artesunate in the Thai-Myanmar border region [61]. Thus, the determination of $p f m d r 1$ gene amplification might become an appropriate tool for the assessment of parasite susceptibility in Ghana [52]. 
The majority of isolates with two copies of the $p f m d r 1$ gene also carried the wild-type N86Y allele compared to a few only harbouring the mutant allele. This suggests that the wild-type allele has a selective advantage to be amplified, although the mutant allele can also have its gene amplified [58].

The PfMDR1 haplotype YFD has been reported to be associated with increased parasite susceptibility to artemisinin derivatives, mefloquine, halofantrine and lumefantrine $[22,62,63]$. The high frequency of this haplotype in Lambaréné suggests that it contributes to maintain parasite susceptibility to artemisinin derivatives, compared to Kumasi and Kisumu where the haplotype was found at low frequencies. The $p f m d r 1$ NFD haplotype identified at higher prevalences in Kumasi and Kisumu than in Lambaréné might decrease parasite susceptibility to AL as it appears to be selected by AL $[43,64,65]$. Therefore, it must be well monitored in countries using AL [52], as the wild-type allele N86Y and potentially its duplication are associated with decreased parasite susceptibility to AL [36, 44]. Moreover, Malmberg et al. [49] showed that NFD followed by the other NYD, YYY and YYD haplotypes was significantly linked with resistance to higher lumefantrine concentration in vivo in Tanzania.

The pfatp6 L263E and S769N mutations have been shown to reduce by allelic exchange parasite susceptibility to some artemisinins $[16,66]$ and to increase the IC50 of artemether [19]. These variants were not observed in these samples. L263E was not present in these isolates compared to the Greater Mekong subregion [67] though identified in field isolates from Tanzania after ACT were introduced [68]. Described as a potential molecular marker for artemisinin resistance [19], the S769N mutation was not present in this study population. Numerous studies have reported this mutation to be rare or absent [67, 69-75]. However, when assessed in African isolates, this mutation did not correlate with an increased IC50 to dihydroartemisinin, the main active compound of artemisinin derivatives [76] as investigated with the increased IC50 of artemether in isolates from French Guiana [19, 69, 77]. An explanation could be that either the functionality of this mutation is only linked to artemether, or that the distinct genetic makeup of the isolate plays a role [76], or that the frequency is still too low to allow for reliable conclusions. H243Y was found with low frequencies in the present study. However, its role in modulating artemisinin resistance is not well characterized [76]. The polymorphism E431K, reported by Jambou et al. to be linked with an increased artemether IC50 [19], was the most prevalent in this study. A previous study has reported an increased frequency of the A623E mutation after the implementation of ACT in Niger [75], while its prevalence in this study was low.
The $p f k 13$ is a polymorphic gene located $5.9 \mathrm{~kb}$ upstream of the $35 \mathrm{~kb}$ region. It is linked with delayed parasite clearance [78] and within the region of topranked signatures of selection on chromosome 13 [79]. Therefore, it has been proposed to be a reliable marker for artemisinin resistance [25]. Among the polymorphisms investigated by Ariey et al. [25] the M476I variant was acquired in vitro in the F32-ART5 $P$. falciparum lineage following increased concentrations of artemisinin. This polymorphism was absent in these isolates, suggesting that the development of artemisinin resistance in the field might differ in vivo and in vitro. The polymorphisms Y493H, R539T and C580Y, associated with a significant slow-clearing of parasites were also absent in this study. Even E612D found in Gambia [80] was absent in these isolates, again indicating the enormous heterogeneity of malaria parasites in Africa. The absence of these $p f k 13$ mutations in African isolates highlights two facts: (1) the mechanisms of artemisinin resistance in Africa are not identical with those observed in Southeast Asia and (2) artemisinin resistance occurring in Cambodia and Thailand has not spread to Africa yet.

Artemisinin and its derivatives remain highly efficacious in the treatment of malaria, including in Africa. The declining efficacy of ACT (TFACT) reported in Southeast Asia threatens the global efforts towards the elimination and eradication of the disease. Selective pressure on the multidrug-resistance gene favoured the return of chloroquine-sensitive (wild-type pfmdr1 N86Y allele) strains as observed in this study and supported by accumulated evidences from previous studies [81, 82]. However, these alleles have been associated with decreased lumefantrine sensitivity. Furthermore, a re-emergence and persistence of $P$. falciparum isolates with multicopies of the $p f m d r 1$ gene in some parts of Africa were noted. This study indicates the need to continuously monitor parasite susceptibility to artemisinin and its derivatives in field isolates from malaria-endemic countries.

\section{Abbreviations \\ PFMDR1: Plasmodium falciparum multidrug resistance 1; PfATP6: Plasmodium falciparum $\mathrm{Ca}^{2+}$-ATPase; PfK13: Plasmodium falciparum Kelch-13 propeller domain; CNV: copy number variation; ACT: artemisinin-based combination therapy; IC50: half maximum inhibitory concentration; SMAC: Severe Malaria in African children; WHO: World Health Organization.}

\section{Authors' contributions}

TPV designed the experiments, supervised the experiments, performed data analysis and wrote the manuscript. AAA, TA, BRO are involved in patient recruitment from respective study sites and are principal investigators for SMAC study. PGK and SK contributed to study design and materials. CNN performed the experiments, data analysis and drafted the manuscript. All authors read and approved the final manuscript.

\section{Author details}

${ }^{1}$ Institute of Tropical Medicine, University Tübingen, Wilhelmstrasse 27 72074 Tübingen, Germany. ${ }^{2}$ Fondation Congolaise pour la Recherche 
Médicale, Brazzaville, Republic of Congo. ${ }^{3}$ Centre de Recherches Médicales de Lambaréné, Lambaréné, Gabon. ${ }^{4}$ Department of Physiology, University of Science and Technology, School of Medical Sciences, Kumasi, Ghana. ${ }^{5}$ Departments of Child Health and Medicine, Komfo Anokye Teaching Hospital, Kumasi, Ghana. ${ }^{6}$ Centre for Clinical Research, Kenya Medical Research Institute, Kisumu, Kenya. ${ }^{7}$ Institute for Infection and Immunity, St George's University of London, London, UK. ${ }^{8}$ Vietnamese-German Center for Medical Research, Hanoi, Vietnam. ${ }^{9}$ Faculty of Medicine, Duy Tan University, Da Nang, Vietnam.

\section{Acknowledgements}

We would like to thank the staff and technicians of Lambaréné, Kumasi and Kisumu for their assistance in collecting the blood samples and clinical data of patients during the study. We express our gratitude to all the study participants involved in this study. We also acknowledge Velia Grummes for technical help during sequencing procedures. We thank the Severe Malaria in African Children (SMAC) consortium.

\section{Competing interests}

The authors declare that they have no competing interests.

\section{Availability of data and materials}

All relevant data are within the paper.

\section{Consent for publication}

All authors read and approved to its final submission.

\section{Ethics approval and consent to participate}

Written informed consent was obtained from parents or guardians of all participating children. The study was approved by the authorities of each study site: the Regional Ethics Committee in Lambaréné (CERIL) for Gabon, the Committee on Human Research, Publication and Ethics, Kwame Nkrumah University of Science and Technology (KNUST), Kumasi for Ghana, and the National Ethics Research Committee, Kenya Medical Research Institute (KEMRI) for Kenya.

\section{Funding}

This work was funded by a DFG grant (DFG-KU775/17-1) German-African cooperation project on infectious diseases. The authors acknowledge the support by the Deutsche Forschungsgemeinschaft (DFG) and Open Access Publishing Fund of Tübingen University. The work is a part of the SMAC study (Trial registry; PACTR201102000277177) funded by European and Developing Countries Clinical Trials Partnership (EDCTP; CT.2004.31070.001). The authors also acknowledge the support from Central African Network on Tuberculosis, and HIV/AIDS (CANTAM).

\section{Publisher's Note}

Springer Nature remains neutral with regard to jurisdictional claims in published maps and institutional affiliations.

Received: 2 February 2017 Accepted: 17 May 2017

Published online: 23 May 2017

\section{References}

1. WHO. Guidelines for the treatment of malaria. 2nd ed. Geneva: World Health Organization; 2010.

2. Dondorp AM, Nosten F, Yi P, Das D, Phyo AP, Tarning J, et al. Artemisinin resistance in Plasmodium falciparum malaria. N Engl J Med. 2009;361:455-67.

3. Amaratunga C, Sreng S, Suon S, Phelps ES, Stepniewska K, Lim P, et al. Artemisinin-resistant Plasmodium falciparum in Pursat province, western Cambodia: a parasite clearance rate study. Lancet Infect Dis. 2012;12:851-8.

4. Phyo AP, Nkhoma S, Stepniewska K, Ashley EA, Nair S, McGready R, et al. Emergence of artemisinin-resistant malaria on the western border of Thailand: a longitudinal study. Lancet. 2012;379:1960-6.
5. WHO. Global plan for artemisinin resistance containment (GPARC). Geneva: World Health Organization; 2011.

6. WHO. Global report on antimalarial drug efficacy and drug resistance: 2000-2010. Geneva: World Health Organization; 2010.

7. Flegg JA, Guerin PJ, White NJ, Stepniewska K. Standardizing the measurement of parasite clearance in falciparum malaria: the parasite clearance estimator. Malar J. 2011;10:339.

8. White NJ. The parasite clearance curve. Malar J. 2011;10:278

9. Kyaw MP, Nyunt MH, Chit K, Aye MM, Aye KH, Aye MM, et al. Reduced susceptibility of Plasmodium falciparum to artesunate in southern Myanmar. PLOS ONE. 2013;8:e57689.

10. Noedl H, Se Y, Schaecher K, Smith BL, Socheat D, Fukuda MM. Artemisinin resistance in Cambodia 1 study C. Evidence of artemisinin-resistant malaria in western Cambodia. N Engl J Med. 2008;359:2619-20.

11. Krishna S, Kremsner PG. Antidogmatic approaches to artemisinin resistance: reappraisal as treatment failure with artemisinin combination therapy. Trends Parasitol. 2013;29:313-7.

12. Krishna S, Staines HM, Kremsner PG. Artemisinin resistance and the blame game. Clin Infect Dis. 2016;63:1144-5.

13. Meshnick S. Artemisinin resistance in southeast Asia. Clin Infect Dis. 2016;63:1527.

14. Hastings IM, Kay K, Hodel EM. The importance of scientific debate in the identification, containment, and control of artemisinin resistance. Clin Infect Dis. 2016;63:1527-8.

15. Eckstein-Ludwig U, Webb RJ, Van Goethem ID, East JM, Lee AG, Kimura $M$, et al. Artemisinins target the SERCA of Plasmodium falciparum. Nature. 2003;424:957-61.

16. Uhlemann AC, Cameron A, Eckstein-Ludwig U, Fischbarg J, Iserovich P, Zuniga FA, et al. A single amino acid residue can determine the sensitivity of SERCAs to artemisinins. Nat Struct Mol Biol. 2005;12:628-9.

17. Cowman AF, Karcz S. Drug resistance and the P-glycoprotein homologues of Plasmodium falciparum. Semin Cell Biol. 1993;4:29-35.

18. Duraisingh MT, Jones P, Sambou I, von Seidlein L, Pinder M, Warhurst DC. The tyrosine-86 allele of the pfmdr1 gene of Plasmodium falciparum is associated with increased sensitivity to the anti-malarials mefloquine and artemisinin. Mol Biochem Parasitol. 2000;108:13-23.

19. Jambou R, Legrand E, Niang M, Khim N, Lim P, Volney B, et al. Resistance of Plasmodium falciparum field isolates to in vitro artemether and point mutations of the SERCA-type PfATPase6. Lancet. 2005;366:1960-3.

20. Price RN, Uhlemann AC, van Vugt M, Brockman A, Hutagalung R, Nair S, et al. Molecular and pharmacological determinants of the therapeutic response to artemether-lumefantrine in multidrug-resistant Plasmodium falciparum malaria. Clin Infect Dis. 2006;42:1570-7.

21. Uhlemann AC, Krishna S. Antimalarial multi-drug resistance in Asia: mechanisms and assessment. Curr Top Microbiol Immunol. 2005;295:39-53.

22. Uhlemann AC, McGready R, Ashley EA, Brockman A, Singhasivanon P, Krishna S, et al. Intrahost selection of Plasmodium falciparum pfmdr I alleles after antimalarial treatment on the northwestern border of Thailand. J Infect Dis. 2007;195:134-41.

23. Reed MB, Saliba KJ, Caruana SR, Kirk K, Cowman AF. Pgh1 modulates sensitivity and resistance to multiple antimalarials in Plasmodium falciparum. Nature. 2000;403:906-9.

24. Duraisingh MT, Cowman AF. Contribution of the pfmdr1 gene to antimalarial drug-resistance. Acta Trop. 2005;94:181-90.

25. Ariey F, Witkowski B, Amaratunga C, Beghain J, Langlois AC, Khim N, et al. A molecular marker of artemisinin-resistant Plasmodium falciparum malaria. Nature. 2014;505:50-5.

26. Amaratunga C, Witkowski B, Khim N, Menard D, Fairhurst RM. Artemisinin resistance in Plasmodium falciparum. Lancet Infect Dis. 2014;14:449-50.

27. Ashley EA, Dhorda M, Fairhurst RM, Amaratunga C, Lim P, Suon S, et al. Spread of artemisinin resistance in Plasmodium falciparum malaria. N Engl J Med. 2014;371:411-23.

28. Huang F, Takala-Harrison S, Jacob CG, Liu H, Sun X, Yang H, et al. A Single mutation in $\mathrm{K} 13$ predominates in Southern China and is associated with delayed clearance of Plasmodium falciparum following artemisinin treatment. J Infect Dis. 2015;212:1629-35.

29. Takala-Harrison S, Jacob CG, Arze C, Cummings MP, Silva JC, Dondorp AM, et al. Independent emergence of artemisinin resistance mutations among Plasmodium falciparum in Southeast Asia. J Infect Dis. 2015;211:670-9.

30. Greenwood B, Mutabingwa T. Malaria in 2002. Nature. 2002;415:670-2. 
31. Pulcini S, Staines HM, Pittman JK, Slavic K, Doerig C, Halbert J, et al. Expression in yeast links field polymorphisms in PfATP6 to in vitro artemisinin resistance and identifies new inhibitor classes. J Infect Dis. 2013;208:468-78.

32. Straimer J, Gnadig NF, Witkowski B, Amaratunga C, Duru V, Ramadani AP, et al. Drug resistance. K13-propeller mutations confer artemisinin resistance in Plasmodium falciparum clinical isolates. Science. 2015;347:428-31.

33. Kremsner PG, Adegnika AA, Hounkpatin AB, Zinsou JF, Taylor TE, Chimalizeni $Y$, et al. Intramuscular artesunate for severe malaria in African children: a multicenter randomized controlled trial. PLoS Med. 2016;13:e1001938.

34. Humphreys GS, Merinopoulos I, Ahmed J, Whitty CJ, Mutabingwa TK, Sutherland CJ, et al. Amodiaquine and artemether-lumefantrine select distinct alleles of the Plasmodium falciparum mdr 1 gene in Tanzanian children treated for uncomplicated malaria. Antimicrob Agents Chemother. 2007;51:991-7.

35. Zakeri S, Hemati S, Pirahmadi S, Afsharpad M, Raeisi A, Djadid ND. Molecular assessment of atpase 6 mutations associated with artemisinin resistance among unexposed and exposed Plasmodium falciparum clinical isolates to artemisinin-based combination therapy. Malar J. 2012;11:373.

36. Price RN, Uhlemann AC, Brockman A, McGready R, Ashley E, Phaipun $\mathrm{L}$, et al. Mefloquine resistance in Plasmodium falciparum and increased pfmdr1 gene copy number. Lancet. 2004;364:438-47.

37. Livak KJ, Schmittgen TD. Analysis of relative gene expression data using real-time quantitative PCR and the $2-\triangle \triangle C T$ method. Methods. 2001:25:402-8.

38. Nguetse CN, Meyer CG, Adegnika AA, Agbenyega T, Ogutu BR, Kremsner $P G$, et al. Glucose-6-phosphate dehydrogenase deficiency and reduced haemoglobin levels in African children with severe malaria. Malar J. 2016;15:346.

39. Nguetse CN, Kremsner PG, Velavan TP. FOXO3A regulatory polymorphism and susceptibility to severe malaria in Gabonese children. Immunogenetics. 2015;67:67-71.

40. Koukouikila-Koussounda F, Ntoumi F, Ndounga M, Tong HV, Abena AA, Velavan TP. Genetic evidence of regulatory gene variants of the STAT6, IL10R and FOXP3 locus as a susceptibility factor in uncomplicated malaria and parasitaemia in Congolese children. Malar J. 2013;12:9.

41. Velavan TP, Buyukyazici B, Kremsner PG, Kun JF. Combined promoter haplotypes of the IL10R genes are associated with protection against severe malaria in Gabonese children. Immunogenetics. 2012;64:87-95.

42. Mayengue PI, Kalmbach Y, Issifou S, Kremsner PG, Ntoumi F. No variation in the prevalence of point mutations in the Pfcrt and Pfmdr1 genes in isolates from Gabonese patients with uncomplicated or severe Plasmodium falciparum malaria. Parasitol Res. 2007;100:487-93.

43. Malmberg M, Ngasala B, Ferreira PE, Larsson E, Jovel I, Hjalmarsson A, et al. Temporal trends of molecular markers associated with artemetherlumefantrine tolerance/resistance in Bagamoyo district, Tanzania. Malar J. 2013;12:103.

44. Sisowath C, Stromberg J, Martensson A, Msellem M, Obondo C, Bjorkman A, et al. In vivo selection of Plasmodium falciparum pfmdr $186 \mathrm{~N}$ coding alleles by artemether-lumefantrine (Coartem). J Infect Dis. 2005;191:1014-7.

45. Kwansa-Bentum B, Ayi I, Suzuki T, Otchere J, Kumagai T, Anyan WK, et al. Plasmodium falciparum isolates from southern Ghana exhibit polymorphisms in the SERCA-type PfATPase6 though sensitive to artesunate in vitro. Malar J. 2011;10:187.

46. Spalding MD, Eyase FL, Akala HM, Bedno SA, Prigge ST, Coldren RL, et al. Increased prevalence of the pfdhfr/phdhps quintuple mutant and rapid emergence of pfdhps resistance mutations at codons 581 and 613 in Kisumu, Kenya. Malar J. 2010;9:338.

47. Veiga MI, Dhingra SK, Henrich PP, Straimer J, Gnadig N, Uhlemann AC, et al. Globally prevalent PfMDR1 mutations modulate Plasmodium falciparum susceptibility to artemisinin-based combination therapies. Nat Commun. 2016;7:11553.

48. Duraisingh MT, Roper C, Walliker D, Warhurst DC. Increased sensitivity to the antimalarials mefloquine and artemisinin is conferred by mutations in the pfmdr1 gene of Plasmodium falciparum. Mol Microbiol. 2000;36:955-61.

49. Malmberg M, Ferreira PE, Tarning J, Ursing J, Ngasala B, Bjorkman A, et al. Plasmodium falciparum drug resistance phenotype as assessed by patient antimalarial drug levels and its association with pfmdr1 polymorphisms. J Infect Dis. 2013;207:842-7.

50. Venkatesan M, Gadalla NB, Stepniewska K, Dahal P, Nsanzabana C, Moriera C, et al. Polymorphisms in Plasmodium falciparum chloroquine resistance transporter and multidrug resistance 1 genes: parasite risk factors that affect treatment outcomes for $P$. falciparum malaria after artemether-lumefantrine and artesunate-amodiaquine. Am J Trop Med Hyg. 2014;91:833-43.

51. Ruetz S, Delling U, Brault M, Schurr E, Gros P. The pfmdr1 gene of Plasmodium falciparum confers cellular resistance to antimalarial drugs in yeast cells. Proc Natl Acad Sci USA. 1996;93:9942-7.

52. Duah NO, Matrevi SA, de Souza DK, Binnah DD, Tamakloe MM, Opoku VS, et al. Increased pfmdr1 gene copy number and the decline in pfcrt and pfmdr1 resistance alleles in Ghanaian Plasmodium falciparum isolates after the change of anti-malarial drug treatment policy. Malar J. 2013;12:377.

53. Sidhu $A B$, Valderramos SG, Fidock DA. pfmdr1 mutations contribute to quinine resistance and enhance mefloquine and artemisinin sensitivity in Plasmodium falciparum. Mol Microbiol. 2005:57:913-26.

54. Tukwasibwe S, Mugenyi L, Mbogo GW, Nankoberanyi S, Maiteki-Sebuguzi C, Joloba ML, et al. Differential prevalence of transporter polymorphisms in symptomatic and asymptomatic falciparum malaria infections in Uganda. J Infect Dis. 2014;210:154-7.

55. Ashley EA, White NJ. Artemisinin-based combinations. Curr Opin Infect Dis. 2005;18:531-6.

56. Uhlemann AC, Ramharter M, Lell B, Kremsner PG, Krishna S. Amplification of Plasmodium falciparum multidrug resistance gene 1 in isolates from Gabon. J Infect Dis. 2005;192:1830-5.

57. Sidhu AB, Uhlemann AC, Valderramos SG, Valderramos JC, Krishna S, Fidock DA. Decreasing pfmdr1 copy number in Plasmodium falciparum malaria heightens susceptibility to mefloquine, lumefantrine, halofantrine, quinine, and artemisinin. J Infect Dis. 2006;194:528-35.

58. Holmgren G, Bjorkman A, Gil JP. Amodiaquine resistance is not related to rare findings of pfmdr1 gene amplifications in Kenya. Trop Med Int Health. 2006;11:1808-12.

59. Eyase FL, Akala HM, Ingasia L, Cheruiyot A, Omondi A, Okudo C, et al. The role of Pfmdr1 and Pfcrt in changing chloroquine, amodiaquine, mefloquine and lumefantrine susceptibility in western-Kenya P. falciparum samples during 2008-2011. PLoS ONE. 2013;8:e64299.

60. Witkowski B, Nicolau ML, Soh PN, Iriart X, Menard S, Alvarez M, et al. Plasmodium falciparum isolates with increased pfmdr 1 copy number circulate in West Africa. Antimicrob Agents Chemother. 2010;54:3049-51.

61. Na-Bangchang K, Muhamad P, Ruaengweerayut R, Chaijaroenkul W, Karbwang J. Identification of resistance of Plasmodium falciparum to artesunate-mefloquine combination in an area along the Thai-Myanmar border: integration of clinico-parasitological response, systemic drug exposure, and in vitro parasite sensitivity. Malar J. 2013;12:263.

62. Nawaz F, Nsobya SL, Kiggundu M, Joloba M, Rosenthal PJ. Selection of parasites with diminished drug susceptibility by amodiaquine-containing antimalarial regimens in Uganda. J Infect Dis. 2009;200:1650-7.

63. Wurtz N, Fall B, Pascual A, Diawara S, Sow K, Baret E, et al. Prevalence of molecular markers of Plasmodium falciparum drug resistance in Dakar, Senegal. Malar J. 2012;11:197.

64. Thomsen TT, Ishengoma DS, Mmbando BP, Lusingu JP, Vestergaard LS, Theander TG, et al. Prevalence of single nucleotide polymorphisms in the Plasmodium falciparum multidrug resistance gene (Pfmdr-1) in Korogwe District in Tanzania before and after introduction of artemisinin-based combination therapy. Am J Trop Med Hyg. 2011;85:979-83.

65. Thomsen TT, Madsen LB, Hansson HH, Tomas EV, Charlwood D, Bygbjerg IC, et al. Rapid selection of Plasmodium falciparum chloroquine resistance transporter gene and multidrug resistance gene-1 haplotypes associated with past chloroquine and present artemether-lumefantrine use in Inhambane District, southern Mozambique. Am J Trop Med Hyg. 2013;88:536-41.

66. Valderramos SG, Scanfeld D, Uhlemann AC, Fidock DA, Krishna S. Investigations into the role of the Plasmodium falciparum SERCA (PfATP6) L263E mutation in artemisinin action and resistance. Antimicrob Agents Chemother. 2010;54:3842-52.

67. Miao M, Wang Z, Yang Z, Yuan L, Parker DM, Putaporntip C, et al. Genetic diversity and lack of artemisinin selection signature on the Plasmodium falciparum ATP6 in the Greater Mekong Subregion. PLOS ONE. 2013;8:e59192. 
68. Chilongola J, Ndaro A, Tarimo H, ShedrackT, Barthazary S, Kaaya R, et al. Occurrence of pfatpase6 single nucleotide polymorphisms associated with artemisinin resistance among field isolates of Plasmodium falciparum in north-eastern Tanzania. Malar Res Treat. 2015;2015:279028.

69. Jambou R, Martinelli A, Pinto J, Gribaldo S, Legrand E, Niang M, et al. Geographic structuring of the Plasmodium falciparum sarco(endo) plasmic reticulum Ca2 + ATPase (PfSERCA) gene diversity. PLoS ONE. 2010;5:e9424.

70. Tanabe K, Zakeri S, Palacpac NM, Afsharpad M, Randrianarivelojosia M, Kaneko A, et al. Spontaneous mutations in the Plasmodium falciparum sarcoplasmic/endoplasmic reticulum Ca2+ -ATPase (PfATP6) gene among geographically widespread parasite populations unexposed to artemisinin-based combination therapies. Antimicrob Agents Chemother. 2011;55:94-100

71. Tahar R, Ringwald P, Basco LK. Molecular epidemiology of malaria in Cameroon. XXVIII. In vitro activity of dihydroartemisinin against clinical isolates of Plasmodium falciparum and sequence analysis of the P. falciparum ATPase 6 gene. Am J Trop Med Hyg. 2009;81:13-8.

72. Zhang G, Guan Y, Zheng B, Wu S, Tang L. No PfATPase6 S769N mutation found in Plasmodium falciparum isolates from China. Malar J. 2008;7:122

73. Afonso A, Hunt P, Cheesman S, Alves AC, Cunha CV, do Rosario V, Cravo P. Malaria parasites can develop stable resistance to artemisinin but lack mutations in candidate genes atp6 (encoding the sarcoplasmic and endoplasmic reticulum (a2 + ATPase), tctp, mdr1, and cg10. Antimicrob Agents Chemother. 2006;50:480-9.

74. Mugittu K, Genton B, Mshinda H, Beck HP. Molecular monitoring of Plasmodium falciparum resistance to artemisinin in Tanzania. Malar J. 2006;5:126.

75. Ibrahim ML, Khim N, Adam HH, Ariey F, Duchemin JB. Polymorphism of PfATPase in Niger: detection of three new point mutations. Malar J. 2009;8:28.
76. Cojean S, Hubert V, Le Bras J, Durand R. Resistance to dihydroartemisinin. Emerg Infect Dis. 2006;12:1798-9.

77. Legrand E, Volney B, Meynard JB, Mercereau-Puijalon O, Esterre P. In vitro monitoring of Plasmodium falciparum drug resistance in French Guiana: a synopsis of continuous assessment from 1994 to 2005. Antimicrob Agents Chemother. 2008;52:288-98.

78. Cheeseman IH, Miller BA, Nair S, Nkhoma S, Tan A, Tan JC, et al. A major genome region underlying artemisinin resistance in malaria. Science. 2012;336:79-82.

79. Takala-Harrison S, Clark TG, Jacob CG, Cummings MP, Miotto O, Dondorp AM, et al. Genetic loci associated with delayed clearance of Plasmodium falciparum following artemisinin treatment in Southeast Asia. Proc Natl Acad Sci USA. 2013;110:240-5.

80. Amambua-Ngwa A, Tetteh KK, Manske M, Gomez-Escobar N, Stewart LB, Deerhake ME, et al. Population genomic scan for candidate signatures of balancing selection to guide antigen characterization in malaria parasites. PLoS Genet. 2012;8:e1002992.

81. Lucchi NW, Komino F, Okoth SA, Goldman I, Onyona P, Wiegand RE, et al. In vitro and molecular surveillance for antimalarial drug resistance in Plasmodium falciparum parasites in Western Kenya reveals sustained artemisinin sensitivity and increased chloroquine sensitivity. Antimicrob Agents Chemother. 2015:59:7540-7.

82. Mekonnen SK, Aseffa A, Berhe N, Teklehaymanot T, Clouse RM, Gebru T, et al. Return of chloroquine-sensitive Plasmodium falciparum parasites and emergence of chloroquine-resistant Plasmodium vivax in Ethiopia. Malar J. 2014;13:244.

\section{Submit your next manuscript to BioMed Central and we will help you at every step:}

- We accept pre-submission inquiries

- Our selector tool helps you to find the most relevant journal

- We provide round the clock customer support

- Convenient online submission

- Thorough peer review

- Inclusion in PubMed and all major indexing services

- Maximum visibility for your research

Submit your manuscript at www.biomedcentral.com/submit 